\title{
Branchio-oto-renal syndrome-a report of three cases and review of literature
}

\begin{abstract}
Branchio-oto-renal syndrome (BOR) is an autosomal dominant disorder characterized by hearing loss, congenital malformations of ear, branchial arch anomalies and structural or functional anomalies of kidneys. We report a rare clinical scenario of a family of three members with features of BOR syndrome.
\end{abstract}

Keywords: branchial arch anomalies, renal anomalies, BOR , syndromic hearing loss

\author{
Volume 10 Issue 4 - 2018 \\ Shahul Hameed CP, Ravi Ramalingam, \\ Ramalingam KK, Wasim Khan SI, Steive \\ George \\ KKR ENT Hospital \& Research Centre, India
}

\begin{abstract}
Correspondence: Ravi Ramalingam, KKR ENT Hospital \& Research Centre, No. 274 Poonamallee High Road, Chennai, India 6000 I0, India, Tel +9|-44-264 | |444, Fax 9|-44-264I 2727, Email kkrenthospital@gmail.com
\end{abstract}

Received: April 19, 2017 | Published: July 02, 2018
Abbreviations: BOR, branchio-oto-renal syndrome; $\mathrm{ABC}$, absolute bone conduction test, PTA, pure tone audiogram; OME, otitis media; RFT, renal function test; USG, ultra sono gram; TFT, tuning fork tests; KUB, kidneys, ureters and bladder; BERA, brainstem evoked response audiometry; EAC, external auditory canal

\section{Introduction}

Branchio-oto-renal (BOR) syndrome is an autosomal dominant ${ }^{1}$ form of inherited disorder considered as the most common cause of syndromic hearing loss with high penetrance and variable expressivity, characterized by hearing loss, pre-auricular pits, congenital auricular malformations, branchial and renal anomalies. ${ }^{2}$ Most common genetic abnormality seen in BOR is EYA1 gene mutation that maps chromosome 8q13.3. ${ }^{3}$ Three members of a family, who presented with clinical features of BOR syndrome, is being reported here.

\section{Case presentation}

A family from Agartala, India (Figure 1) visiting KKR ENT Hospital and Research Institute, Chennai since 2014 with various ENT complaints was evaluated by complete physical examinations and investigations including audiological tests, renal functions tests and ultrasound of kidneys, ureters and bladder (KUB) for symptoms and signs of BOR.

\section{Patient one}

$55 \mathrm{yr}$ old male with history of difficulty in hearing, swellings in front of the right ear and swellings on both sides of the neck, since birth. He was diagnosed with bilateral branchial cyst and underwent excision of the same in his childhood and biopsy was reported as branchial cyst. He was also diagnosed with bilateral fixed malleus syndrome and underwent left ossiculoplasty in 1997 with no hearing improvement.

On examination, an accessory auricle (Figure 2A-C) was noted on the right side. Pinna on both sides was abnormally protruding, suggestive of a bat ear / otapostasis and 'low set' ears (Figure 2A). Rinne's test was negative bilaterally, Webers was not lateralized and a reduced absolute bone conduction $(A B C)$ bilaterally. Neck examination showed a surgical scar (Figure 2B) of branchial cyst excision on both side of the neck approximately $3.5 \mathrm{~cm}$ below the angle of mandible.

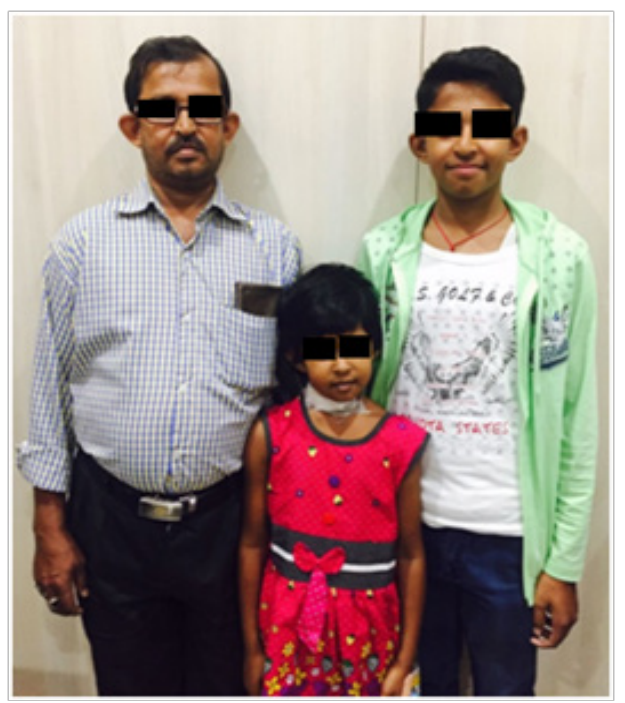

Figure I The family.

Audiological evaluation (Figure 3A \& B) confirmed moderately severe mixed hearing loss on both sides. Ultrasound abdomen and KUB showed few cysts in both kidneys with largest measuring $1.7 \mathrm{x}$ $1 \mathrm{~cm}$ in the lower pole of the right kidney (Figure 3A). Renal function tests (RFT) were normal (urea: 20, creatinine: 0.9).

\section{Patient two}

10 year old boy, son of patient one, was brought with complaints of decreased hearing both ears of 3 years duration associated with history of recurrent cough, cold and mouth breathing. There was also a history of frequent pus discharge from a small opening on the left side of his neck since birth.

Ear examination showed accessory auricles on right side (Figure 4A) and a pre-auricular sinus on left side (Figure 4B) with bilateral 
congenital Bat ear deformity and low set ears (Figure 5A). On otoscopy, bilateral TM showed features suggestive of otitis media with effusion. TFT revealed a negative Rinne's test bilaterally, Webers was not lateralised and a reduced $\mathrm{ABC}$ bilaterally. He had enlarged adenoids and grade II palatine tonsils.
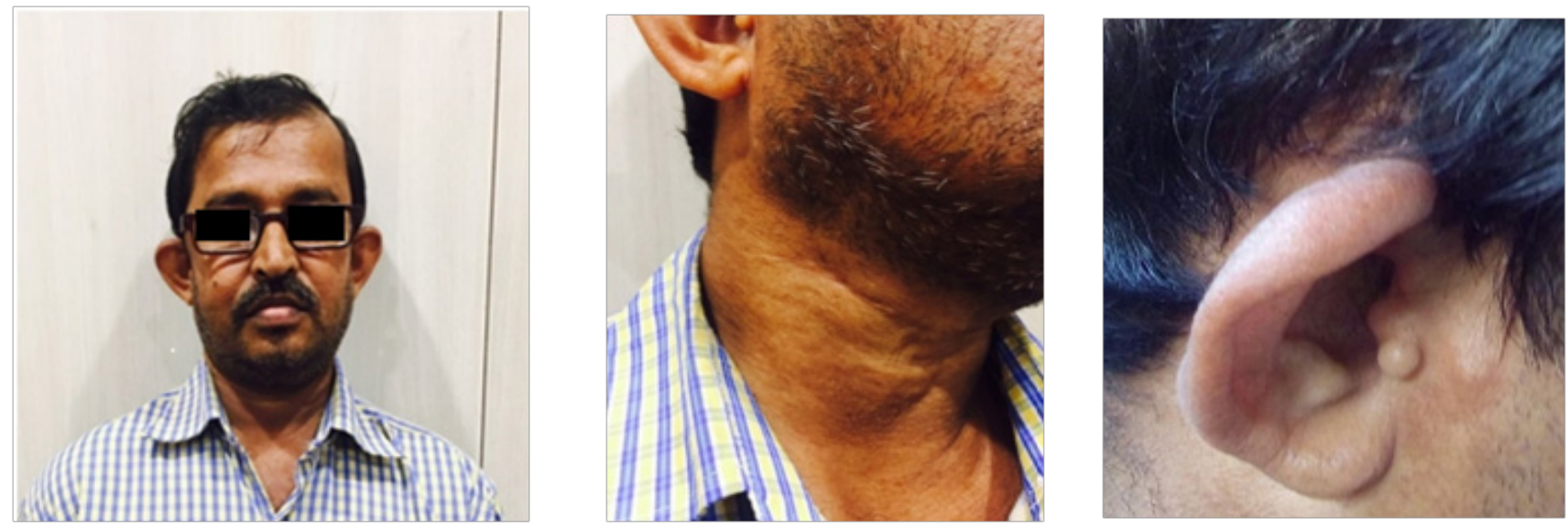

Figure 2A-C Neck examination showed a surgical scar of branchial cyst excision on both side of the neck approximately $3.5 \mathrm{~cm}$ below the angle of mandible.
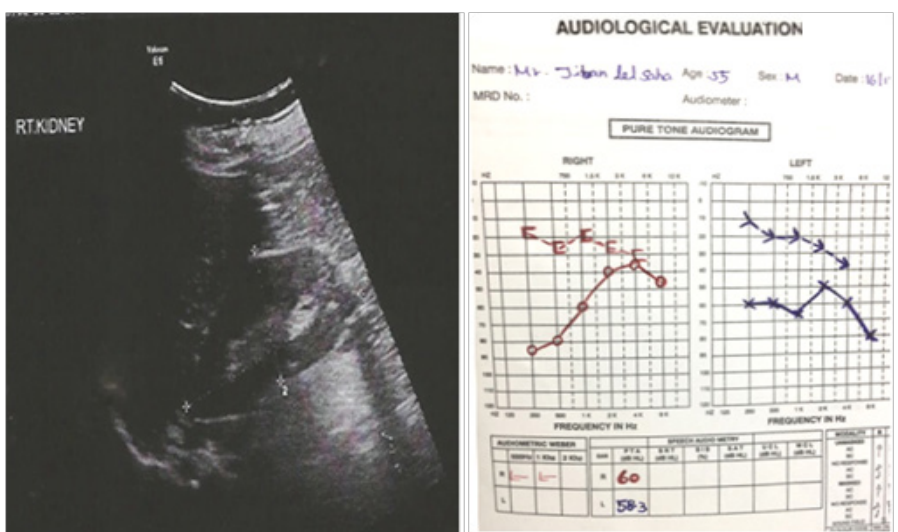

Figure 3A \& B Audiological evaluation confirmed moderately severe mixed hearing loss on both sides.
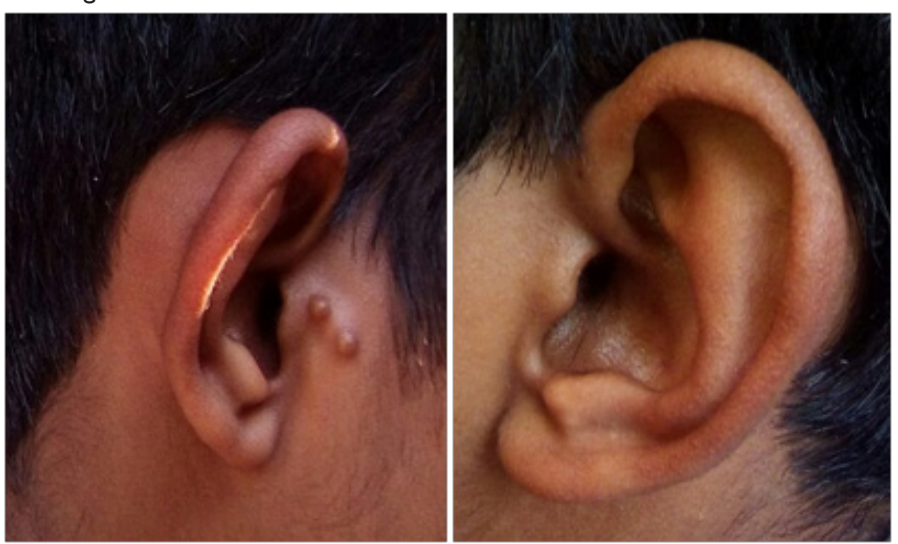

Figure 4A \& B Ear examination showed accessory auricles on right side and a pre-auricular sinus on left side.

On neck examination, there was a small opening in the left side of neck, $3 \mathrm{~cm}$ below the angle of mandible with discharging pus, suggestive of branchial fistula (Figure 5B). Lateral radiograph of the neck confirmed adenoid hypertrophy. PTA showed moderate conductive hearing loss on the right side with severe mixed hearing loss on the left (Figure 5C). Impedence audiometry (IA) showed "B" type tympanogram bilaterally. A diagnosis of chronic adenotonsillitis with bilateral OME with bilateral mixed hearing loss and bilateral congenital external ear malformations along with a left sided branchial fistula was made.

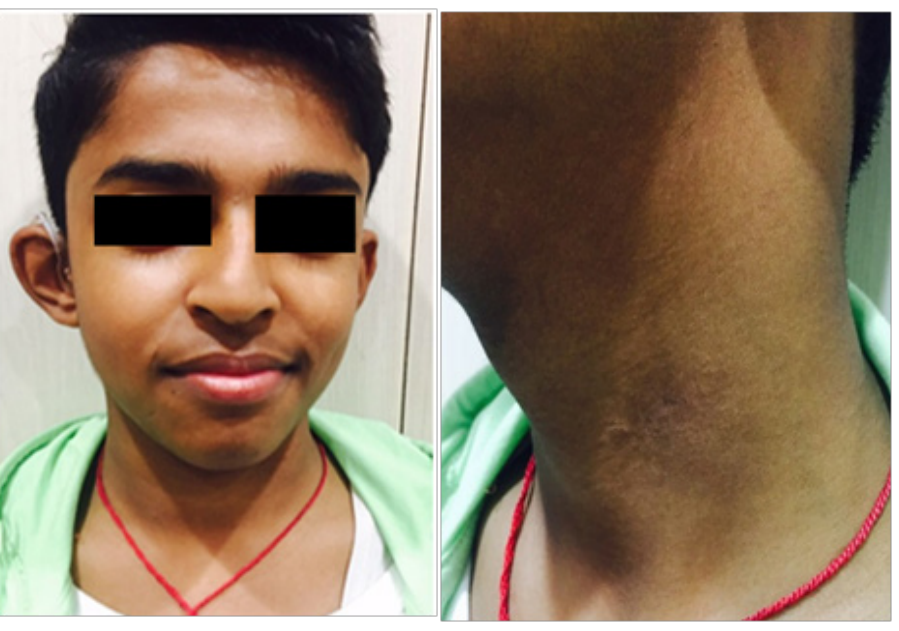

Figure 5A-C PTA showed moderate conductive hearing loss on the right side with severe mixed hearing loss on the left.

$\mathrm{He}$ was treated with adeno-tonsillectomy with bilateral myringotomy (which showed glue in right middle ear with serous fluid on left side) and excision of left branchial fistula.

He was evaluated again in 2016 and the above clinical and audiological findings were found to be the same. His renal evaluation with RFT and USG were normal.

\section{Patient three}

5 year old girl, daughter of patient one, was brought in 2014 with complaints of delayed development of speech and swelling on the right side of neck of 6 months duration. 
On examination, there was a small well defined $2 \times 2 \mathrm{~cm}$ swelling on the right side of neck, $2 \mathrm{~cm}$ below the angle of mandible, oval in shape, non tender, along with a small fistulous opening on the anterior border of sternocleidomastoid muscle at the junction of its upper 2/3rd and lower 1/3rd. ENT examination showed bilateral dull TM and adeno-tonsillar hypertrophy. OAE test was showing PASS. BERA was reported as "hearing sensitivity within normal limits", and IA gave "A" curve on both sides. A speech evaluation done showed her to have an inadequate speech and language development for her age. She was diagnosed with chronic adeno-tonsillitis with right branchial cyst with fistula and was advised an adenotonsillectomy with branchial fistula excision and speech therapy. But, for various reasons, the family opted for speech therapy sessions alone.

She was brought to our hospital again in 2016 with complaints of hearing difficulty and a recurrent history of pus discharge from the swelling on the right side of neck (Figure 6A \& B). Her examination showed Rinne's negative on both sides, Webers, not lateralized and a normal ABC. Her PTA showed bilateral moderately severe conductive hearing loss with IA showing bilateral" B"type tympanogram.
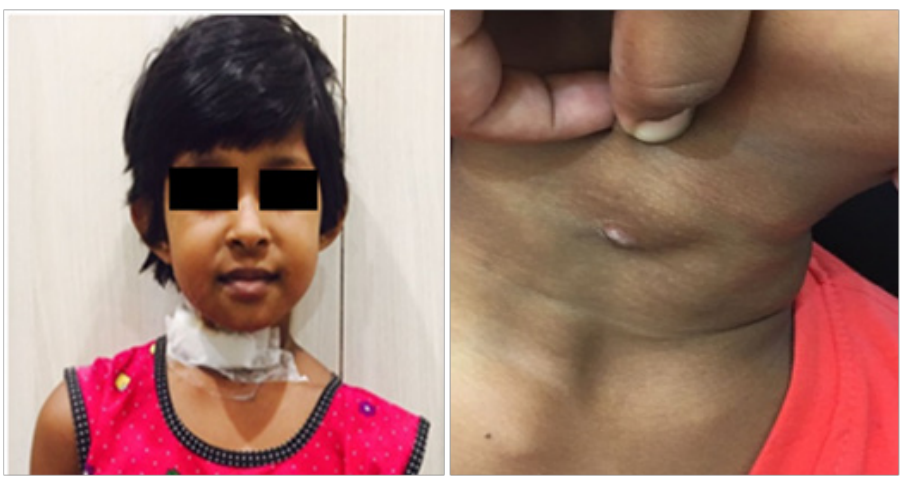

Figure 6A \& B swelling on the right side of neck.

A diagnosis of right branchial fistula with chronic adenotonsillitis with bilateral OME with bilateral conductive hearing loss was made and she underwent an adenotonsillectomy with bilateral myringotomy (thick glue on both sides) with branchial fistula excision. The biopsy confirmed it to be a branchial cyst. Her renal evaluation with RFT and USG were also normal.

\section{Discussion}

Branchio-oto-renal syndrome is an autosomal dominant (Figure 7) form of inherited disorder, considered as the most common syndromic hearing $\operatorname{loss}^{2}$ with high penetrance and variable expressivity characterized by a conductive, sensory neural or mixed hearing loss, pre auricular pits, auricular malformations of outer ear, middle ear or inner ear, branchial fistulae, cysts or sinuses and renal anomalies.

Familial occurance of branchial anomalies were first recognized by Aucherson in 1832, The combination of pre-auricular pits, branchial fistula and hearing impairment was reported by Heusinger in 1864 and Paget in 1877. BOR was referred as 'Hereditory hardness of hearing' by Fourman and Fourman ${ }^{4}$ in 1955. Melnick et al., ${ }^{5}$ in 1975 is credited with the description and addition of renal abnormalities to complete the whole spectrum of BOR underscoring the phenotypic anomalies of branchial arches, otocysts and renal primodia.

Various synonyms for BOR include ear pit-deafness syndrome, pre auricular pits-cervical fistula-hearing loss syndrome, branchio- oto-renal dysplasia, branchio-oto-ureteral syndrome, BOR, BOS, and Melnick-Fraser Syndrome.

\section{Autosomal dominant}

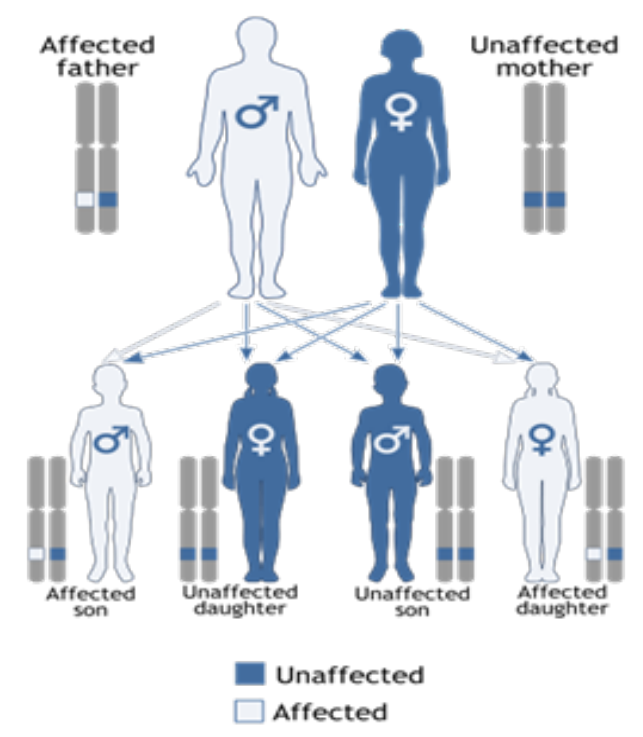

Figure 7 Branchio-oto-renal syndrome is an autosomal dominant.

The triad of BORS is hearing loss with pre auricular fistulae, branchial fistulae and various renal anomalies; ${ }^{1,9}$

Hearing loss: Most common feature, seen in $80 \%$ carriers. It can be non-progressive or progressive, congenital or late onset. Hearing loss may be sensorineural $(20 \%)$, conductive $(30 \%)$ or mixed $(50 \%)$, may range from mild to profound hearing loss. The type of hearing loss may even differ in each ear within an individual. ${ }^{1}$

Other otologic findings include lop ear deformity, pre-auricular cartilaginous appendages, microtia or anotia, EAC stenosis, low set ears and a more subtle finding is the presence of pre-auricular pits. There may be malformations of middle ear and inner ear which is seen on a CT or surgical exploration. ${ }^{6}$

Branchial arch anomalies: Seen in 69\% cases, considered as hallmark feature, can be unilateral or bilateral. It can be congenital or late onset branchial cysts, sinuses or fistulae. ${ }^{2}$

Renal malformations: Spectrum ranges from mild to severe with majority being minor. Only $10 \%$ will have clinically significant renal involvement. Severe abnormalities include bilateral renal agenesis, polycystic kidneys or enlarged blunded kidneys. Others include vesicoureteral reflex, unilateral agenesis, bifid renal pelvis and diverticuli. Renal functional status ranges from normal to chronic renal failure. ${ }^{3}$

Other features: Which may be associated with BOR include nasolacrimal duct anomalies, gustatory lacrimation, facial anomalies, mandibular anomalies, hemi facial microsmia, congenital $7^{\text {th }}$ nerve palsy, ocular manifestations (strabismus or epibulbar dermoid) or $4^{\text {th }}$ and $6^{\text {th }}$ arch anomalies. ${ }^{1}$

There are some major and minor criteria for the diagnosis of BOR (Table 1). The criteria to diagnose BOR syndrome are:

a. The presence of two major deficiencies or the combination of two 
major and two minor anomalies or a major anomaly associated with the presence of another first degree family member diagnosed with the syndrome. ${ }^{2}$

Table I Major and minor criteria for diagnosing BOR

\begin{tabular}{clll}
\hline Major criteria & \multicolumn{2}{c}{ Minor criteria } \\
\hline a. & Second branchial arch & a. & External auditory canal \\
anomalies & & anomalies \\
b. Deafness & b. & Middle ear anomalies \\
c. Preauricular pits & c. & Inner ear anomalies \\
d. Auricular & d. & Preauricular tags \\
& e. & Other: facial asymmetry, \\
e. Renal anomalies & & palate abnormalities
\end{tabular}

Prevalence of BOR is $1: 40,000-1: 70,000^{7}$ with most common pattern of penetrance being autosomal dominant with few showing mitochondrial inheritance and some with 'new' mutations. The first region identified on genetic studies was 8q12-22. Subsequently, after detailed studies, it was concluded that chromosomal region $8 \mathrm{q} 13.3$ is associated with the syndrome. The mutation of EYA1 gene (responsible for development of branchial arches, auditory system and kidneys) has been reported in most cases of BOR syndrome. Missense mutation of SIX1 gene on chromosome 14q23.1 was also reported by several studies. However many cases of clinically diagnosed BOR was negative for above mentioned mutations indicating genetic heterogeneity of BORS. ${ }^{9}$

\section{Conclusion}

BORS is a rare cause of syndromic hearing loss with the phenotypic anomalies of branchial arches, otocysts and renal primodia. Deafness linked to preauricular fistula, branchial fistulae should be investigated and monitored by a multi professional team. Few familial cases have been reported in journals but not many from India. In this study, almost all the phenotypic features of BOR were observed in our first subject. Subjects two and three had some features except renal change. The family has been counseled for a detailed evaluation including intravenous pyelogram (IVP), high resolution CT temporal bone, ${ }^{10}$ genetic study etc. and the need for serial monitoring of the children as they are at a higher risk of developing renal issues later in life.

\section{Acknowledgements}

The authors thank the family that participated in this study.

\section{Conflict of interest}

None.

\section{References}

1. Coppage KB, Smith RJH. Branchio-oto-renal syndrome. J Am Acad Audiol. 1995;6:103-110.

2. Lindau TA, Cardoso ACV, Rossi NF, et al. Anatomical changes and audiological profile in branchio-oto-renal syndrome: a literature review. Int Arch Otolaryngol. 2014118:68-76.

3. Pierides AM, Athanasiou Y, Demetriou K, Koptides M (2002) A family with the branchio-oto-renal syndrome: clinical and genetic correlation. Nephro Dial Transplant. 2002;17(6):1014-1018.

4. Fourman P, Fourman J. Hereditory deafness in a family with ear pits (fistula auriscongenita). Br Med J. 1955;2(4952):1354-1356.

5. Melnick M, Bixler D, Nance WE, et al. Familial branchio-oto-renal dysplasia; a new addition to the branchial arch syndromes. Clin Genet. 1976;9(1):25-34.

6. Fraser FC, Ling D, Clogg D, et al. Genetic aspects of the brancho-otorenal syndrome. Am J Med Genet. 1978;2:241-252.

7. Fraser FC, Sproul JR, Halal F. Frequency of brancho-oto-renal (BOR) syndrome in children with profound hearing loss. Am J Med Genet. 1980;7(3):341-349.

8. Chang EH, Menezes M, Meyer NC, et al. Branchio-oto-renal syndrome: the mutation spectrum in the EYA1 and its phenotypic consequences. Hum Mutat. 2004;23:582-589.

9. Chen A, Francis M, Ni L Cremers CW, et al. Phenotypic manifestations of branchio-oto-renal syndrome. Am J Med Genet. 19995;58:365-370.

10. Ceruti S, Cremers S, Casselman JW. Temporal bone anomalies in the branchio-oto-renal syndrome: detailed computed tomographic and magnetic resonance imaging findings. Oto Neurotol. 2002;23:200-207. 